\title{
Analysis of the Difficulties of the Earth Integrated Information Network
}

\author{
Ding Ying, Yu Hai-yang, Jiang Hui-lin, Hu Yuan, Zhu Yi-feng, Cong Li-gang \\ Key Laboratory of Education Ministry Optoelectronics Measurement \& Control and Optical Information Transfer \\ Technology \\ Changchun University of Science and Technology \\ Changchun, China \\ E-mail: dingying@cust.edu.cn; custhaiyang@126.com;
}

\begin{abstract}
Due to the progress of the satellite communication system, the demands of earth integrated information network become rapid development. In this paper, we researched on the developing history and trend of the space network, and enumerated some important characteristics and difference between the space and territorial networks. And then analyzed the difficulties of the space internet, and proposed some solutions. It hopes to provide a useful reference for the development of the earth integrated information network.
\end{abstract}

Keywords-earth integrated information network; technology difficulty; space-based network; terrestrial networks

\section{INTRODUCTION}

After the C. Clark announced the theory that only three satellites can achieve the global communication, a lot of institutes and universities research the satellite communication system with heart and soul, and some countries own their space-based platform such as satellites and space station. However, nowadays, all the satellite is circuit based not IP, so the connectivity is point-to-point or multi-points which cannot be full mesh at the network layer. With the rapid development of computer and Internet, the integrated between the space-based network and the terrestrial networks will become possible. It will help human to apperceive the resource and environment, and support the service of space infrastructure. It also can be use for the other planets' exploring. Therefore, it is essential to develop the earth integrated network.

The earth integrated network includes the inter-satellite in the space, the plane in the sky, the base station and all kinds of moving terminal. According to the intelligent highspeed processing, packet switching in space, routing technology, it is an integrated network which can accurate access information and transmit information effectively [1]. It's a huge project for the human beings in the aspect of communication, navigation, position, remote sensing, and so on [2].

This paper emphasizes the hard working and the challenge that we will be up against, in order to provide the useful reference for the final implementation of the earth integrated network.

\section{INTERPLANETARY INTERNET OVERVIEW}

The U.S. Defense Advanced Research Projects Agency (DARPA) funded the Jet Propulsion Laboratory (JPL) to launch the study of interplanetary Internet in 1998, and it proposed to extend the terrestrial Internet into space. In the year of 1999, the Iridium System of the Motorola Company broke the original convention of satellite communication system using GEO and transparent transponder communication. Since then, the space-based mobile communication network has gone through a narrow-band satellite communications network, the broadband satellite communications network and the space-based Internet [3].

Most researchers have been fully announced that the earth integrated network play an important role in the military and economic development during the 21st century. Some institutes have made some progress in this field. Some famous space agency, such as the United States NASA, has begun to expand the top-level architecture of this new satellite communication system. In order to meet the needs of future space communications, NASA established a kind of integrated network architecture of communications satellites and other spacecraft in orbit [4](Figure 1).

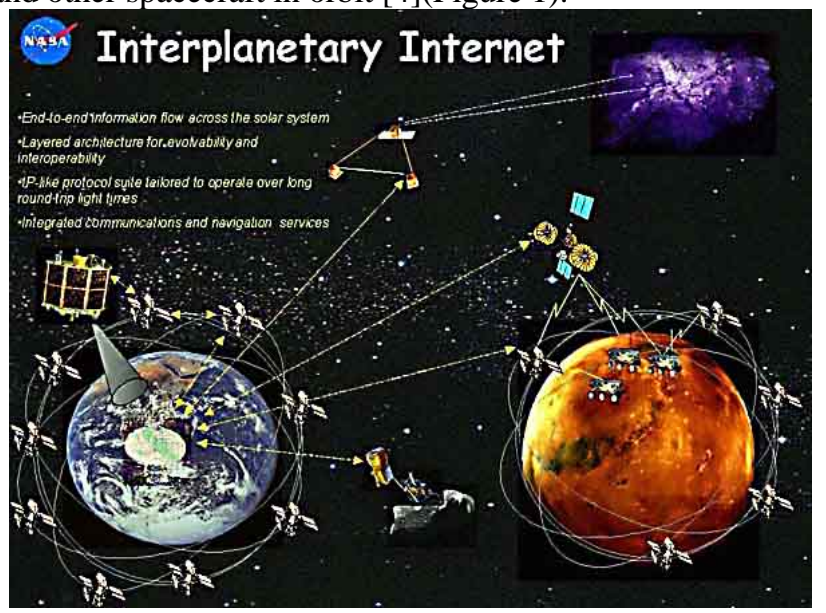

Figure 1. Schematic diagram of interplanetary Internet

Currently, the U.S. has finished the relevant architecture, a preliminary definition of the data format, the Internet Engineering Task Force (IETF) draft agreement and the ongoing demonstration. Meanwhile, the research is continue, just like the dynamic use of space link, the multi-protocol label switching protocol, mobile IP, security issues and how to make each protocol adapted to support space missions.

In 2001, the Goddard Space Center (GSFC) carried out a research project, shown in Figure 2, called "Operating Mission as Nodes on the Internet (OMNI)". It aims to use 
commercial communication protocols on the ground and COTS (commercial off-the-shelf) networking devices, which accord with the ground commercial communications protocol, to achieve standardization of space communications and to verify the feasibility of Internet communication technology for space communications.

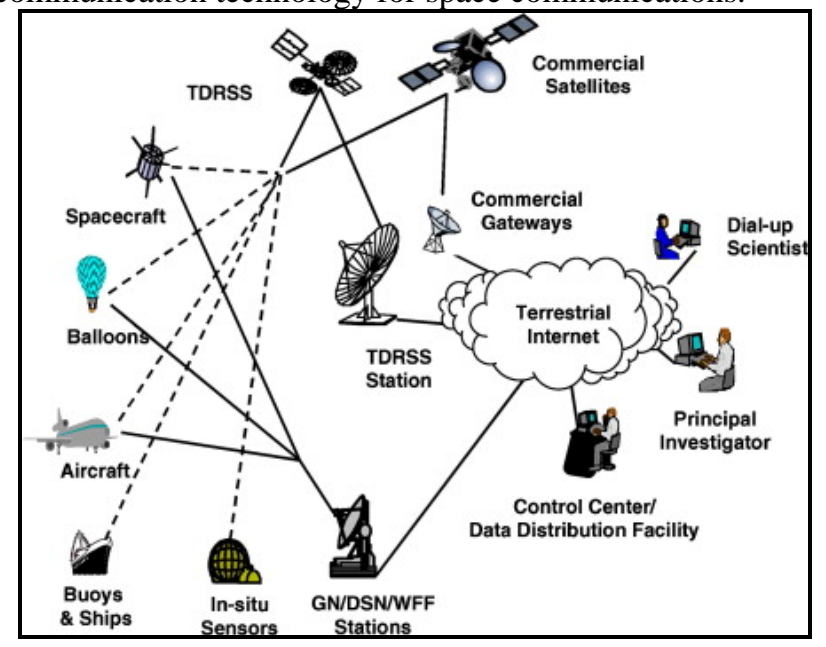

Figure 2. Operating Mission as Modes on the Internet (OMNI)

In 2003, the United States launched the project called" Internet Routing In Space (IRIS)", as shown in Figure 3. First of all, Cisco router is equipped to complete the testing and validation of the key technologies in the United Kingdom UK-the DMC1 LEO satellite. The contents of the validation include: running IPv4 protocol on the CLEO, testing mobile transmission capacity, and demonstrating IPv4 + IPsec and IPv6 protocol. What was more, the router would be equipped to a high orbit satellite of Intelsat 14 in the year of 2009 [5]. Network structure is formed by "space access the main node" and "backbone communication node". This content of the validation include: completed cross-beam routing switching technology testing on the satellite, as well as demonstrated the connecting with other routers by singlehop.

In addition, ESA also developed terrestrial transmission protocol called SLE (Space link extension) which based on the CCSDS (Consultative Committee for Space Data Systems) [6].It has been incorporated into the architecture of CCSDS, so the Space Transfer Protocol of CCSDS will extend to the aerospace facilities on the ground.

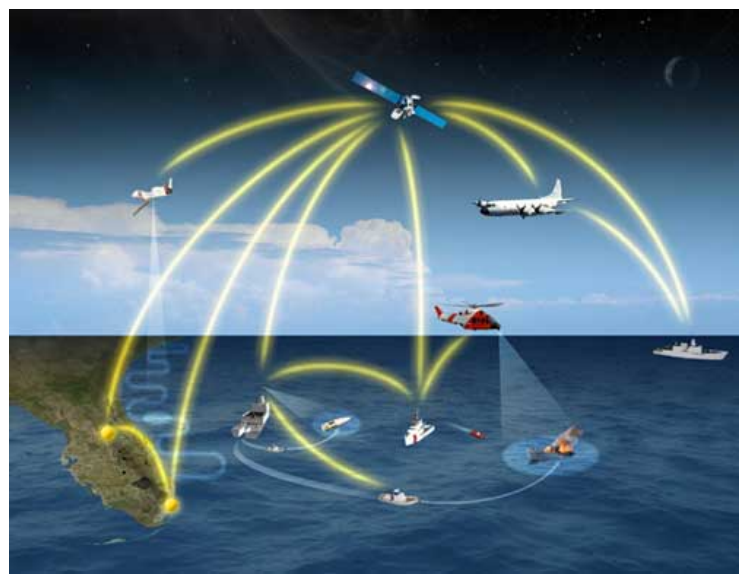

Figure 3. Internet Routing In Space (IRIS)

\section{CHARACTERISTICS AND DIFFICULTIES}

\section{A. Characteristics}

According to the scope of application and the type of communication conditions, compared with the terrestrial network, the space network has its unique characteristics. The main features are reflected in the following aspects:

- Firstly, the network is large scale. Earth integrated network is a three-dimensional information network that constituted by all kinds of network platform, such as the space-based, air-based, terrestrial-based, and other nodes. With the development of industrial technology and space technology, the growth of information types and quantities are accelerated. The satellite constellation system, which constituted by multiple satellites, need to establish the communication link among the satellites, put the switching on the satellites, or to establish a gateway for the exchanging and processing capabilities [7]. Due to the differences in architecture and network protocol of different platforms, such as satellites, aircraft, terminal facilities on the ground, and so on, the complexity to achieving the space-based information network is increased [8]. A satellite and a variety of spacecraft continue to emerge, a variety of aviation, spacecraft orbital parameters and the operation mode are not the same and have different functions. It may vary from a member of the space information network become more diversified.

- Secondly, a wide range of covering. Earth integrated network covers the satellite, approaching spacecraft and the three-dimensional network of land system and sea system. They become a wide range of area. The applications of earth integrated network can increase the coverage area of the terminal. Compared with terrestrial cellular systems, spacebased network is in the high altitude region of space. Earth integrated network has larger coverage angle. Compared to the Internet, it has less number of space network nodes and the relatively simple routing, so it can provide a business of large-span, farther and wider coverage using of multiple access mode transmission of the satellite communications [9]. For example, the mobile phones, the scheduling 
communications, the data communications, the wireless location, the paging services, and so on.

- Thirdly, the network is dynamic and irregular. The topology of the network is a highly dynamic threedimensional structure, and the member nodes have highspeed operation on the same rail, the spatial positional relationship produce a variety of changes among nodes over time, which result in changes of the network topology. The whole network that includes the aviation, aerospace and aircraft is extensively used and has a strong mobility. Due to the decline in the quality of the communication link and communication interruption, it causes the changes in the topology relations and makes network become dynamic and irregular greatly increasing.

- Fourthly, distributed and ad-hoc character is afford. Earth integrated network is a wireless ad-hoc network. The nodes not only play the role of independent routing, but also be the hosts in the network. It composes autonomous systems that support multi-hop at any time at any place. There is no network central control point like base station in the network, all the nodes have the equal status. A node can adopt a distributed approach to coordination and control each other's behavior, thus enhancing the robustness of network.

- Fifthly, the high requirement of terminals is offered. The more limited of the size, weight, power consumption and the shape of the antenna of the mobile terminal device in the installation of the carrier (such as aircraft, cars, ships, etc.), the more stringent requirements for the handheld terminal[10]. Satellite antenna beam should be able to adapt to the ground coverage area change and maintain the orientation pointing, the antenna beam of the user mobile terminal should be able to keep the directivity of the satellite, for the user moves, or it becomes omnidirectional antenna beam. In addition, the working frequency band of a variety of user link among the satellite mobile communication system is restricted, if using radio frequency communication, the frequency is usually between $200 \mathrm{MHz}$ and $10 \mathrm{GHz}$.

- Sixthly, the big delay of extended link and the nodes wobbles exist. Earth integrated network both have synchronous orbit which is about $36,000 \mathrm{~km}$ from the ground, and the planes which is just a few kilometers away from the ground. The distance between nodes is much larger than the distance among the nodes on the ground. The link transmission delay is also larger than the delay on the ground. At the same time, due to the nodes have dynamic operation with high speed, and each node have difference among the relative movement speed between two nodes, the nodes wobbles become bigger. So considering the key technology of group gateway like routing policy, the delay in the terrestrial network which can usually be ignored cannot be ignored in the earth integrated network.

- Finally, quality of network link is seriously affected by the environment. Earth integrated network uses wireless communication basically, and it is vulnerable to the impact of environmental factors in the space. The water in the air, oxygen ions would cause signal power loss in the natural environment. The rain, dust and other inclement weather can affect the signal transmission in the atmosphere. Factors of human beings like fading, collision and blocking can also influence signal interference. As a result, it will lead to the degradation of communication link.

In summary, the above characteristics lead to a theory that the earth integrated network is different from the usual networks. It has larger coverage, wider service, higher utilization, higher real time, strong invulnerability, etc. But for the reasons of constraint of the space applications costs, the limit of function of node and poor security of wireless channel, it faces up to the limited bandwidth and other issues.

\section{B. Difficulties:}

The construction of earth integrated network is different from the terrestrial networks. Owing to the longer distance, less nodes, bigger delay and other characteristics in spacebased systems, it has unique characteristics in technology. Meanwhile, these characteristics add the design complexity of network and the difficulty of realization. They are reflected in the following aspects:

- Firstly, the network is large-scale, three-dimensional structure and composition complexity, so it is difficult to design the architecture.

Earth integrated network covers three-dimensional network of the satellite, near space aircraft and sea-land system covering a wide area. With the development of a variety of aircraft and the increasing number of satellite, the type and quantity of the member nodes continue to grow.

Due to a variety of aviation, the spacecraft orbital parameters and the way of running are not the same, making members of space information network have become more diversification. These make the earth to integrated network structure become very complex, increasing the difficulty of network architecture's design and implementation.

- Secondly, due to irregular network structure and topology structure is changing all the time, it is difficult to achieve router on stars.

In the earth integrated network, the network topology is a perspective view of a highly dynamic structure, the member node in a high-speed operation of the different track, the spatial positional relationship between the attitude of the satellite and the node varies over time, which is result to network topology changed in real time. And aviation, aerospace and aircraft communications platform has great mobility, it is difficult to determine the position of the aircraft next time, these have greatly increased the dynamic and irregular network, thus making the star on the road by the algorithm design is difficult.

- Thirdly, delay of the link is long and the nodes wobble, so it is difficult to manage the network.

There are synchronous orbit satellites, unmanned aerial vehicles and other aircraft in the near-Earth space in the earth integrated network, the distance between nodes is much larger than terrestrial networks, the link transmission delay is much larger than the terrestrial network. Therefore, the transmission delay can usually be ignored in the terrestrial network, but it cannot be ignored in the earth integrated network. Meanwhile, due to the operating status of the node is high dynamic, aircraft and satellite angular velocity change, it is caused by the delay wobbling between nodes. 
Extended transmission delay wobbling and other characteristics increase the difficulty of the routing policy and network management technology.

- Fourthly, network link quality is seriously affected by the environment, so it is difficulty to achieving space exchange.

Earth integration network using wireless communication, volume of traffic on the network link exposed in space, In addition to the effects such as signaling fall, collision, congestion, noise, it also limited by the space environment and air capacity. This makes the actual bandwidth further below the theoretical bandwidth, and the result will cause a reduction in communication link performance and error rate rise. How to improve the adaptability of communication and ratio performance, such as link bandwidth, signal-to-noise and so on, is one of the technical difficulties of earth integration network.

- Fifthly, payload on the satellites and node functionality are limited, so it is difficulty to dealing with processing on the satellites.

As the load limited and other causes communication system memory, CPU processing power, limited electric power, the earth integrated network nodes with wired network nodes have an extremely limited compared to the satellite. The algorithm of dealing with processing on the satellites has many constraints, and the difficulty of processing on the satellites system implementation is greatly increased.

- Sixthly, exposed to the propagation environment and poor security, it is difficulty to confirm the confidentiality of communication.

Earth integrated network is a highly open wireless distributed architecture. It is more vulnerable to touch security threats such as eavesdropping, intrusion, network attacks and denial of service. How to improve the confidentiality and security of the system is one of the technical difficulties.

\section{CONCLUSION}

Building the earth integrated information network is important scientific and strategic significance. It will meet the need of the national army and the people shared in wartime and peaceful time. During the peaceful time, it can play a role for domestic mobile communications, radio, television, distance education and disaster relief. In the war time, it can provide strong support for transmission command of the troops and command military information acquisition. Through the interpretation of foreign development course and the investigation of interpretation domestic related research institution, this paper analyzed and summarized the key technology and technical difficulties needed to be overcome in the integration of earth network construction. The paper also pointed out the current main direction, providing a reference for the network construction of integration of earth network of our country.

Earth integrated network which is scaled largely involves a lot of technical content. With profound research and understanding, we also need to study new principles and new technology of spatial information network.

\section{REFERENCES}

[1] Shen Rong-jun. The mind of earth integrated network and spaceflight [J].China Engineering Science, 2006, 8(10):19-30.

[2] FARSEROTU J,PRASAD R.A.Survey of future broadband multimedia satellite systems, issues and trends[J].IEEE Communications Magazine,2000,(6):128-133.

[3] Kul Bhasin, Jeffrey Hayden. Developing Architectures and Technologies for an Evolvable NASA Space Communication Infrastructure[R]. National Aeronautics and Space Administration: Washington, DC 20546-0001.2004,7.

[4] GUI Qi-shan, ZHAO Xin-guo, GUO Wei-min, GU Xiao-xia. Study on US Army Space Information System Establishment[J]. Network and Information Technology.2008,27 (1) 63-65.

[5] Bhasin K. and Hayden J. . "Space Internet Architectures and Technologies for NASA Enterprises[R]," IEEE Aerospace.Conference, March 2001.

[6] Jingshan Jiang, Guoliang Bian. Space-based Information Network for Earth Observation[R]. Beijing: Science Press, Digital EarthProceedings of the International Symposium on Digital Earth,1999.

[7] Space Communications and Navigation (SCaN) Network Architecture Definition Document (ADD) Volume 1: Executive Summary[R], Revision 2, Oct 2011, NASA / SCaN.

[8] Behnam Malakooti, Ivan J. Thomas, Kul Bhasin and Allen Holtz. A Framework for an Intelligent Internet Protocol for a Space-Based Internet[R]. American Institute of Aeronautics and Astronautics. California, USA, 2004-5.

[9] Stylianos Karapantazis, Fotini-Niovi Pavlidou. QoS Handover Management for Multimedia LEO Satellite Networks [J] Telecommunication Systems,2006,32(4): 225-245.

[10] Peter Shames, Michael Anderson, Steve Kowal, Michael Levesque, Oleg Sindiy, Kenneth Donahue, Patrick Barnes. NASA Integrated Network Monitor and Control Software Architecture. USA. 